\title{
Identificación de parámetros de un modelo térmico para un motor trifásico de inducción usando algoritmos genéticos
}

\section{Parameters identification of a thermal model for a three-phase induction motor using genetic algorithm}

Luis Diego Murillo-Soto', Geovanni Figueroa-Mata², Osvaldo Guerrero-Castro ${ }^{3}$

Fecha de recepción: 19 de junio del 2015 Fecha de aprobación: 26 de setiembre del 2015

Murillo-Soto, L; Figueroa-Mata, G; Guerrero-Castro, O. Identificación de parámetros de un modelo térmico para un motor trifásico de inducción usando algoritmos genéticos. Tecnología en Marcha. Edición especial. Matemática Aplicada, Mayo 2016. Pág 25-41.

1 Profesor Asociado, Máster en Computación, Instituto Tecnológico de Costa Rica, : Imurillo@tec.ac.cr

2 Profesor Catedrático, Máster en Computación,, Instituto Tecnológico de Costa Rica, gfigueroa@tec.ac.cr

3 Profesor Catedrático, Máster en Administración de la Ingeniería Electromecánica,, Instituto Tecnológico de Costa Rica, oguerrero@tec.ac.cr 


\title{
Palabras claves
}

Algoritmo genético; modelo térmico del motor de inducción; identificación.

\section{Resumen}

Una forma alternativa de identificar los parámetros de un modelo térmico de un motor de inducción es por medio de algoritmos genéticos. Este trabajo en su inicio, describe brevemente los conceptos relacionados con los modelos térmicos de motores, así como los principales conceptos de los algoritmos genéticos. Posteriormente plantea un modelo térmico simplificado para un motor trifásico Baldor modelo M3558, de $2 \mathrm{hp}, 230 \mathrm{~V}, 60 \mathrm{~Hz}$, dicho modelo posee dos nodos para los cuales se plantean sus ecuaciones diferenciales y sus soluciones analíticas. Para determinar los parámetros de este modelo térmico se alimenta un algoritmo genético con el cálculo de las pérdidas del motor eléctrico así como las temperaturas del motor capturadas por un banco de pruebas construido para tal fin. El algoritmo genético va modificando cada parámetro de la solución analítica hasta que se cumplan los criterios de ajuste. En la parte final del trabajo se confrontan las capacitancias y conductancias térmicas obtenidas contra cálculos teóricos de las constantes físicas del motor. Se muestra que los parámetros obtenidos con el algoritmo genético presentan un mejor ajuste respecto a los datos y no requieren del conocimiento de las magnitudes y constantes físicas relacionadas con el motor.

\section{Keywords}

Genetic algorithms; induction motor thermal model; parameter identification.

\begin{abstract}
Genetics algorithms are an alternative way to identify the parameters of an induction motor thermal model. Initially, this work briefly describes the concepts related to electric motors thermal models and the key concepts of genetic algorithms. Then it presents a simplified model for an M3558 Baldor motor, $2 \mathrm{HP}, 230 \mathrm{~V}, 60 \mathrm{~Hz}$, the thermal model have two nodes, a set of differential equations and its analytical solutions is derived for each one. To determine the parameters of the thermal model, a genetic algorithm is fed with the calculation of motor losses and temperatures captured by a tests bench. The genetic algorithm changes each parameter of the analytical solution until adjustment criteria are met. At the end of this work, the thermal capacitances and inductances obtained are compared with theoretical calculations of the physical constants of the motor. We demonstrate that the parameters obtained with genetic algorithms fit better to the data and require little knowledge of the magnitude and physical constants related to the motor.
\end{abstract}

\section{Introduccion}

Debido a que los motores eléctricos de inducción representan la principal fuerza motriz para accionar los sistemas electromecánicos, estimar de forma correcta la temperatura interna de la máquina es una necesidad por su efecto sobre la vida útil de la máquina. Los materiales y barnices aislantes del motor se degradan de forma exponencial, por cada $10^{\circ} \mathrm{C}$ por encima de la temperatura máxima de la clase de aislamiento la vida útil se reduce a la mitad (Oraee, 2000; Pillay \& Manyage, 2006).

$$
L_{x}=L_{100} \cdot 2^{(T c-T x) / k}
$$


Donde $L_{x}$ representa la estimación de la vida útil en la nueva condición de operación, $L_{100}$ es la vida útil proyectada de fábrica usualmente 20.000 horas, Tx es la nueva temperatura de operación, Tc es la temperatura de la clase del aislamiento y $k$ es una constante definida para cada clase de aislamiento donde 14, 11, 9.3, 8, y 10 corresponden a los aislantes clase A, B, $\mathrm{F}, \mathrm{H}$ y $\mathrm{H}^{\prime}$.

La estimación de la temperatura interna del motor se puede dividir en cinco grandes estrategias tal y como se muestra en la figura 1.

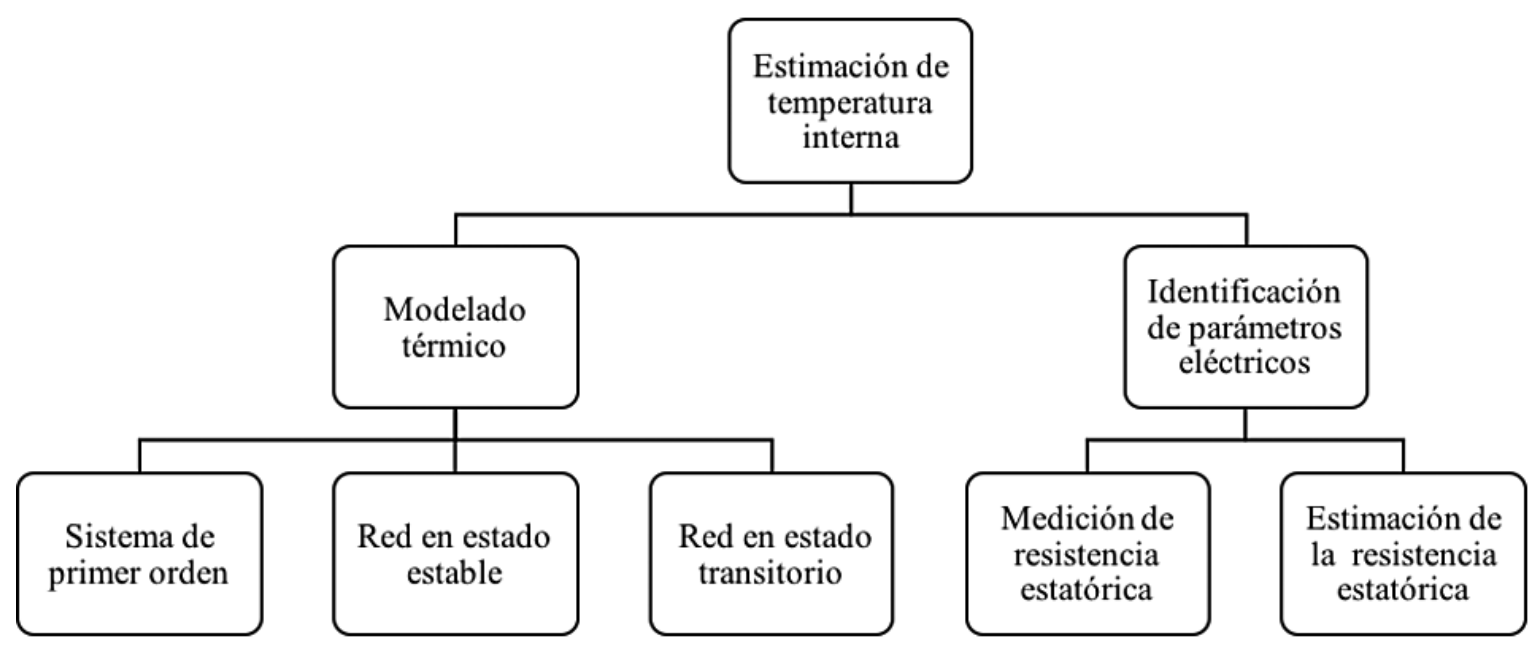

Figura 1. Clasificación de los métodos para estimar temperaturas.

De acuerdo con (Gao, 2006), los modelos de primer orden son rápidos y eficientes, presentan una buena estimación de la temperatura y necesitan solamente la corriente estatórica, pero su estimación es un promedio general que no permite determinar los puntos calientes de la máquina. En (Zocholl, 2004) se realiza una comparación entre distintos tipos de modelos de primer orden, los cuales poseen una estructura similar a la ecuación (2).

$$
\theta=I^{2} R R_{t h}\left(1-e^{\frac{-t}{R_{t h} C_{t h}}}\right)+\theta_{A}
$$

Donde $\theta$ y $\theta_{A}$ son las temperaturas interna y la ambiental respectivamente, el término $R R$ representa la totalidad de pérdidas del motor, $R_{t h}$ representa una resistencia térmica equivalente y $C_{t h}$ representa la capacitancia térmica del motor.

Por su parte, los modelos térmicos mallados para estados estables permiten predecir la temperatura en zonas específicas de la máquina, con el inconveniente de que la estimación de temperaturas en el transitorio térmico presenta errores de hasta el 100\%. Estos modelos en estado estable tales como los que se plantean en (Kylander, 1995; Shenkman \& Chertkov, 2000; Dutta \& Chowdhury, 2012), obtienen las temperaturas resolviendo sistemas de ecuaciones lineales (3), donde $P$ es el vector de pérdidas de la máquina, $G$ es la matriz de conductancias térmicas del motor y $\theta$ es el vector de temperaturas a calcular.

$$
P=G \theta
$$

Los modelos térmicos mallados para estados transitorios son aquellos que pueden predecir la temperatura en un punto específico de la máquina, tanto en régimen transitorio como en estado estable. Estos modelos incorporan un tercer vector a la ecuación (3), el cual se forma al 
multiplicar una matriz $C$ que contiene en su diagonal las capacitancias térmicas de la máquina, por un vector que contiene las derivadas de las temperaturas respecto al tiempo $d \theta / d t$, de tal forma que la expresión general queda:

$$
\boldsymbol{C} \frac{\partial \boldsymbol{\theta}}{\partial t}=\boldsymbol{P}-\boldsymbol{G} \boldsymbol{\theta}
$$

Los modelos complejos que muestran el estado transitorio tal como y como se plantean (Oraee, 2000; Duran \& Fernandez, 2004; Moreno, Hidalgo, \& Martínez, 2001) ofrecen una predicción de la temperatura en el respectivo nodo, pero para poderla estimar se requiere conocer los materiales, geometrías, dimensiones y masas para calcular cada conductancia térmica entre los nodos $G$ y la matriz de capacitancia $C$. Además, es necesario conocer en forma detallada cada una de las pérdidas reales $P$ de la máquina, para eso se debe realizar pruebas de laboratorio. Cabe señalar que estos inconvenientes también se presentan en los modelos en estado estable, ecuación (3).

La estimación de la temperatura con base en parámetros eléctricos, se divide en dos esquemas fundamentales. El primero surge del estándar IEEE 112 ("IEEE Standard Test Procedure for Polyphase Induction Motors and Generators," 2004), en donde se indica que la variación de la temperatura interna provoca variaciones de las resistencias estatóricas y rotóricas del motor en la siguientes proporciones.

$$
\begin{gathered}
R_{2}=R_{1} \frac{\theta_{2}+k}{\theta_{1}+k} \\
\theta_{2}=\frac{R_{2}}{R_{1}}\left(\theta_{1}+k\right)-k
\end{gathered}
$$

Donde $R_{1}$ representa la resistencia a la temperatura $\theta_{1}$, usualmente medido a temperatura ambiente con motor apagado, $R_{2}$ la resistencia a la temperatura $\theta_{2}$ y $k$ es el coeficiente del material conductor, que para el cobre es 234.5 y para el aluminio es de 225. La ecuación (6) muestra el resultado de despejar la temperatura interna. Para obtener el valor de las resistencias se utilizan dos métodos, el primero calcula la resistencia aplicando la ley de Ohm a la medición del voltaje y corriente continua (CD). El segundo es la medición directa con puente wheatstone doble, mediante un miliohmímetro. Consecuentemente y dado que el punto más caliente de una máquina es el devanado estatórico (Boldea \& Nasar, 2002), la medición de la resistencia estatórica es el método más sencillo para la estimación de temperaturas.

La desventaja del esquema de medición de la resistencia estatórica es que es invasivo, ya que requiere de equipamiento adicional y desconectar eléctricamente la máquina. Por tal motivo se han venido desarrollando métodos alternativos de obtención de la resistencia estatórica a partir de mediciones eléctricas de voltajes y corrientes, (Gao, 2006; Gao, Habetler, Harley, \& Colby, 2005) o frecuencias (Cho \& Seok, 2008). La idea de estos trabajos es desarrollar equipos en tiempo real que operen en línea con el motor y que realicen los respectivos cálculos de la estimación de temperatura interna. El principal inconveniente de estos métodos es que estiman la temperatura interna del devanado, pero no estima el comportamiento de temperaturas en otras zonas de interés en el motor, tal como la temperatura del núcleo, roles, carcasa, etc. Por otro lado, si se aplican técnicas metaehurísticas es posible estimar los parámetros de los modelos térmicos (3) y (4) que es la propuesta del presente trabajo.

\section{Algoritmo genético}

Los algoritmos genéticos (GA) fueron desarrollados por John Holland en la década de los años 70. Se fundamentan en los principios biológicos de la evolución de las especies: selección natural y supervivencia del más fuerte.

Son algoritmos poblacionales, ya que trabajan con un conjunto de soluciones factibles llamado población. De este modo, en lugar de pasar de una única solución a la siguiente, se pasa 
de una población a otra mediante la combinación y mutación de soluciones factibles. La combinación de soluciones factibles se lleva a cabo mediante cruzamiento, con la esperanza de producir descendientes mejor adaptados que sus ancestros. De esta manera, la especie evoluciona adaptándose mejor al entorno. Eventualmente, se producen mutaciones con la idea de producir descendientes con características distintas a las de sus progenitores, estas son las causantes de la aparición de individuos superiores. Sin mutaciones la especie no podría evolucionar hacia organismos más complejos y mejor adaptados a su medio. Por esta razón, el proceso de mutación se considera primordial en el proceso de evolución (Talbi, 2009).

La selección de individuos que formarán la nueva generación, buscan imitar la selección natural, en el sentido de que los individuos más adaptados tienen mayor probabilidad de ser seleccionados para formar la nueva generación mediante cruzamiento y mutación.

Existen muchas formas de realizar el proceso de selección. Entre las más usadas están los métodos de selección estocástica como la selección por ruleta simple y la selección por torneo determinístico o probabilístico.

Los algoritmos genéticos tradicionalmente se utilizan resolver problemas de búsqueda y/o optimización, en los cuales se tiene que determinar la mejor solución de un conjunto de soluciones factibles de gran tamaño.

A cada uno de las soluciones factibles de una generación se le asocia un valor llamado aptitud. Este valor cuantifica la calidad de la solución y permite comparar soluciones de la misma generación y/o con soluciones de generaciones anteriores.

Entrada: Parámetros del algoritmo

Salida: Individuo mejor adaptado (mejor solución)

1: $t \leftarrow 0$

2: Generar la población inicial $P_{t}$, con $m$ individuos

3: Mientras no se haya satisfecho el criterio de parada

4: Calcular la aptitud de cada individuo en $P_{t}$

5: $P_{t} \leftarrow \operatorname{Cruzar}\left(P_{t}\right)$

6: $P_{t} \leftarrow \operatorname{Mutar}\left(P_{t}\right)$

7: $t \leftarrow t+1$

8: Construir la nueva población $P_{t}$

9: fin

10: Retornar la mejor solución obtenida.

Figura 2. Algoritmo genético básico.

El algoritmo genético comienza con una población inicial de soluciones factibles, que se construye usualmente de forma aleatoria. Se evalúa la aptitud de cada individuo de la población y se construye nueva población mediante cruzamiento, mutación y selección. Este proceso se repite hasta que se satisfaga algún criterio de parada, como se muestra en la figura 2. 
Con respecto a la convergencia de un algoritmo genético, Rudolph (Rudolph, 1994), demuestra que las variantes de un algoritmo genético básico que mantengan en la población, antes o después de la selección, la mejor solución hallada a lo largo del tiempo, siempre converge al óptimo global. Esto no se puede garantizar cuando se utiliza la selección elitista pues el mejor individuo no sólo se mantiene sino que también se utiliza para generar nuevos individuos y el algoritmo tiene una matriz de transición diferente y, por tanto, diferentes dinámicas de búsqueda que pueden ser mejores en algunos casos y peores en otros.

\section{Modelo Térmico Propuesto}

El modelo térmico que se utiliza para el motor de estudio fue propuesto por (Duran y Fernandez, 2004; Moreno, Hidalgo y Martínez, 2001). Este modelo se construye a partir de la ecuación general (4). El modelo de la figura 3, posee sólo dos nodos llamados $\theta_{s}$ y $\theta_{r}$ que representan la temperatura promedio del estator y del rotor respectivamente. Por otra parte, la temperatura $\theta_{0}$ se refiere a la temperatura ambiental. El modelo representa las pérdidas de potencia del motor como fuentes de calor que elevan las temperaturas internas. La fuente del estator es llamada $P_{s}$ y la del rotor $P_{r}$. Los capacitores térmicos $C_{s}$ y $C_{r}$ representan la capacidad del estator y el rotor de almacenar calor, son el producto de la masa del componente por el calor específico del material.

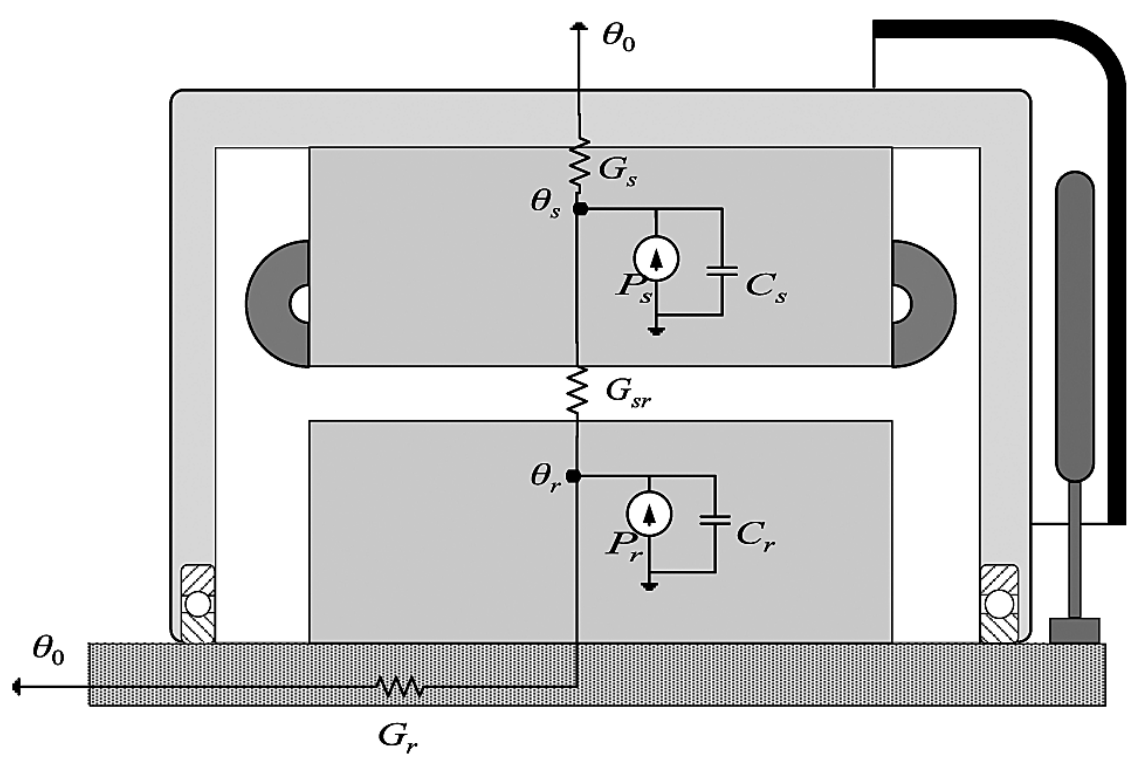

Figura 3. Modelo térmico de un motor eléctrico de inducción. Adaptado de Duran y Fernandez, (2004).

\section{Estimación de las pérdidas}

El incremento de temperatura dentro de un motor de inducción es causado por la energía que no se logra transformar por unidad de tiempo y se desperdicia como calor, es decir las pérdidas son resultado de un deficiente proceso de conversión de energía eléctrica a mecánica. Por lo tanto, la pérdida total $P_{T}$ es la diferencia de potencia de la entra $P_{E n t}$ menos la potencia de salida $P_{\text {Sal }}$ de la máquina. Estas pérdidas usualmente se agrupan en tres clases: las llamadas pérdidas fijas $P_{f}$, las pérdidas variables $P_{v}$ y pérdidas misceláneas $P_{s t}$ 


$$
P_{T}=P_{\text {Ent }}-P_{\text {Sal }}=P_{f}+P_{v}+P_{s t}
$$

Las pérdidas fijas $P_{f}$ son aquellas que se asocian a las pérdidas en el núcleo del motor $P_{N}$ más las pérdidas mecánicas $P_{M}$, ecuación (8). La pérdida $P_{N}$ se compone de la pérdida por histéresis $P_{H}$ más la pérdida que producen las corrientes parásitas $P_{F}$. Las pérdidas mecánicas $P_{M}$ se componen a su vez de las pérdidas por fricción en rodamientos $P_{R}$ y por las pérdidas por vendaval $P_{v}$. De acuerdo con Fraile-Mora (2008) las potencia por pérdida en núcleo y mecánica se modelan como :

$$
\begin{gathered}
P_{f}=P_{N}+P_{M} \\
P_{N}=P_{H}+P_{F}=\left(k_{H} f B_{m}{ }^{\alpha}+k_{F} f^{2} B_{m}{ }^{2} a^{2} \sigma\right) v \\
P_{M}=P_{R}+P_{V}=A \omega+B \omega^{3}
\end{gathered}
$$

Para la ecuación (9) las constantes $k_{H}$ y $k_{F}$ representan los efectos de histéresis y de Foucault respectivamente, $f$ es la frecuencia, $B_{m}$ es el campo magnético máximo, los parámetros $\alpha, a$, $\sigma, v$ representan las contantes de diseño: el espesor de placas, la conductividad y volumen del hierro respectivamente. Para la ecuación (10) $A, B$ son contantes y $\omega$ representa la velocidad del eje.

Dado que en la ecuación (9) todos los parámetros son constantes a excepción de la frecuencia de funcionamiento y el campo magnético, pero estos son prácticamente constantes, entonces se dice que la potencia $P_{N}$ es constante en la curva de bajo deslizamiento. Eventualmente el contenido armónico de la señal de voltaje incrementa estas pérdidas, ya que existirán frecuencias múltiplos de $f$ que generan campos magnéticos residuales que se suman a las pérdidas provocada en la frecuencia fundamental. De igual forma sucede con las pérdidas mecánicas (10), la velocidad de régimen es prácticamente constante, y varía muy poco respecto a la velocidad en vacío. Las pérdidas fijas se obtienen aplicando la prueba en vacío que aparece en la norma IEEE 112 ("IEEE Standard Test Procedure for Polyphase Induction Motors and Generators" 2004), y la ecuación siguiente para cada voltaje:

$$
P_{f}=P_{E n t 0}-P_{C u 0}
$$

donde $P_{\text {Ento }}$ es el consumo de potencia del motor cuando este no posee carga en el eje y $P_{\text {Cuo }}$ se refiere a las pérdidas por efecto Joule de la resistencia por fase $R_{e}$ del devanado estatórico, de tal forma que para un sistema que presenta corrientes con desbalance se puede calcular como:

$$
P_{C u 0}=R_{e}\left(I_{A 0}^{2}+I_{B 0}^{2}+I_{C 0}^{2}\right)
$$

donde $I_{A 0}, I_{B O}, I_{C O}$ se refieren a las corrientes en vacío de cada una de las fases.

Las pérdidas variables $P_{V}$ son todas aquellas que dependen del porcentaje de carga del motor y por tanto de la corriente que pasa por los conductores. Estas pérdidas son producto del efecto Joule en la resistencia del devanado estatórico $P_{\text {CuE, }}$ así com o en las barras del rotor $P_{\text {CuR. }}$.

$$
P_{C u E}=R_{e}\left(I_{A}{ }^{2}+I_{B}{ }^{2}+I_{C}{ }^{2}\right)
$$

donde $R_{e}$ se refiere a la resistencia a temperatura ambiente y $I_{A}, I_{B}$, $I_{C}$ a las corrientes por fase. La potencia de pérdida del rotor $P_{\text {CuR }}$ se calcula como:

$$
P_{C u R}=P_{E n t}-P_{\text {Sal }}-P_{C u E}-P_{f}-P_{s t}
$$

donde $P_{\text {st }}$ se refiere a la potencia miscelánea que según la norma "IEEE Standard Test Procedure for Polyphase Induction Motors and Generators" (2004) se estima como el 1.8\% de la potencia 
de salida para máquinas menores a 90kW. Otra forma de estimar esta potencia es conociendo el deslizamiento en el punto de operación de la máquina y la potencia de pérdida del núcleo $P_{N}$. Para máquinas de 4 polos la pérdidas mecánicas $P_{M}$ no superan el $1.5 \%$ de la potencia de salida según Kim, Choi, Chun, Koo, y Han, (2014), por lo que la potencia de pérdida del rotor se puede calcular como,

$$
P_{C u R}=\left(P_{E n t}-P_{C u E}-\left(P_{f}-P_{M}\right)\right) s
$$

De acuerdo con el modelo planteado en la sección anterior, la potencia que se pierde en el estator $P_{s}$ y en el rotor $P_{r}$ se calculan como:

$$
\begin{gathered}
P_{S}=\left(P_{C u E}+\left(P_{f}-P_{M}\right)\right) \\
P_{r}=P_{C u R}
\end{gathered}
$$

Deducción del modelo

A partir de la ecuación (4) y del modelo térmico de la figura 3, se obtiene el sistema de ecuaciones diferenciales de primer orden, con sus condiciones iniciales:

$$
\begin{gathered}
{\left[\begin{array}{cc}
C_{s} & 0 \\
0 & C_{r}
\end{array}\right]\left[\begin{array}{c}
\frac{d \theta_{s}}{d t} \\
\frac{d \theta_{r}}{d t}
\end{array}\right]=\left[\begin{array}{l}
P_{s} \\
P_{r}
\end{array}\right]-\left[\begin{array}{cc}
\left(G_{s}+G_{s r}\right) & -G_{s r} \\
-G_{s r} & \left(G_{r}+G_{s r}\right)
\end{array}\right]\left[\begin{array}{l}
\theta_{s} \\
\theta_{r}
\end{array}\right]} \\
\theta(0)=\left[\begin{array}{l}
0 \\
0
\end{array}\right]
\end{gathered}
$$

Donde dichas condiciones significan que el motor se encuentra a temperatura ambiente, es decir $\theta_{s}(0)=0$ y $\theta_{r}(0)=0$.

Se solucionó de forma analítica la ecuación (18) y se obtuvieron las ecuaciones (19) que modelan la temperatura del estator $\theta_{s}$ y del rotor $\theta_{r}$ en función de los parámetros térmicos.

$$
\begin{gathered}
\theta_{s}=C_{1} e^{r_{1} t}+C_{2} e^{r_{2} t}+\frac{E}{B} \\
\theta_{r}=C_{3} e^{r_{1} t}+C_{4} e^{r_{2} t}+\frac{F}{B}
\end{gathered}
$$

donde las constantes de (19) en función de los parámetros son:

$$
\begin{array}{cc}
C_{1}=\frac{E\left(r_{2} C_{s}+G_{s}+G_{s r}\right)-F G_{s r}}{B C_{s}\left(r_{1}-r_{2}\right)} & C_{2}=\frac{E\left(r_{1} C_{s}+G_{s}+G_{r s}\right)-F G_{r s}}{B C_{s}\left(r_{2}-r_{1}\right)} \\
C_{3}=\frac{C_{1}\left(r_{1} C_{s}+G_{s}+G_{s r}\right)}{G_{s r}} & C_{4}=\frac{C_{2}\left(r_{2} C_{s}+G_{s}+G_{s r}\right)}{G_{s r}} \\
B=\frac{G_{s} G_{r}+G_{s} G_{s r}+G_{r} G_{s r}}{C_{s} C_{r}} & E=\frac{G_{r} P_{s}+G_{s r} P_{s}+G_{s r} P_{r}}{C_{s} C_{r}} \\
F=\frac{G_{s} P_{r}+G_{s r} P_{r}+G_{s r} P_{s}}{C_{s} C_{r}} & \text { Donde } r_{1}, r_{2} \text { son las soluciones de la ecuación } \\
\text { caracteristica asociada. }
\end{array}
$$




\section{Banco de pruebas y adquisición de datos}

El banco de pruebas para motores trifásicos se compone de un generador de corriente directa de $5 \mathrm{~kW}$ y un banco de resistencias de $7 \mathrm{~kW}$. El banco posee tres autotransformadores monofásicos conectados en estrella para provocar bajos y altos voltajes de alimentación así como desbalances, finalmente poleas y fajas para acoplar mecánicamente el motor al generador tal como se aprecia en la figura $4 a$.

Se ha desarrollado un software (Murillo-Soto, 2014) que gestiona el banco de pruebas y somete los motores a experimentos controlados en los que se definen los parámetros de la prueba. Se controlan los estímulos del motor (carga, tiempos de encendido o apagado), se monitorea su reacción y finalmente se registran todas las variables de interés.

La aplicación informática se desarrolló desde el paradigma de programación gráfica usando LabView v12.0. La aplicación posee una arquitectura cliente-servidor punto a punto y se compone de dos programas uno que se ejecuta en una computadora personal y el otro que se ejecuta en un controlador de tiempo real C-RIO 9073 de National Instruments, tal como se muestra en la figura 4b. Los siete módulos que tiene instalado el C-RIO instalados son: un NI-9227 para corrientes trifásicas, un NI-9225 para voltajes trifásicos, un NI-9422 para entradas digitales, un NI-9478 para salidas digitales, un NI-9207 para entradas analógicas de voltaje, un NI-9263 para salidas analógicas, un módulo NI-9219 universal para RTD y un NI-9213 para termopilas j y $\mathrm{k}$.

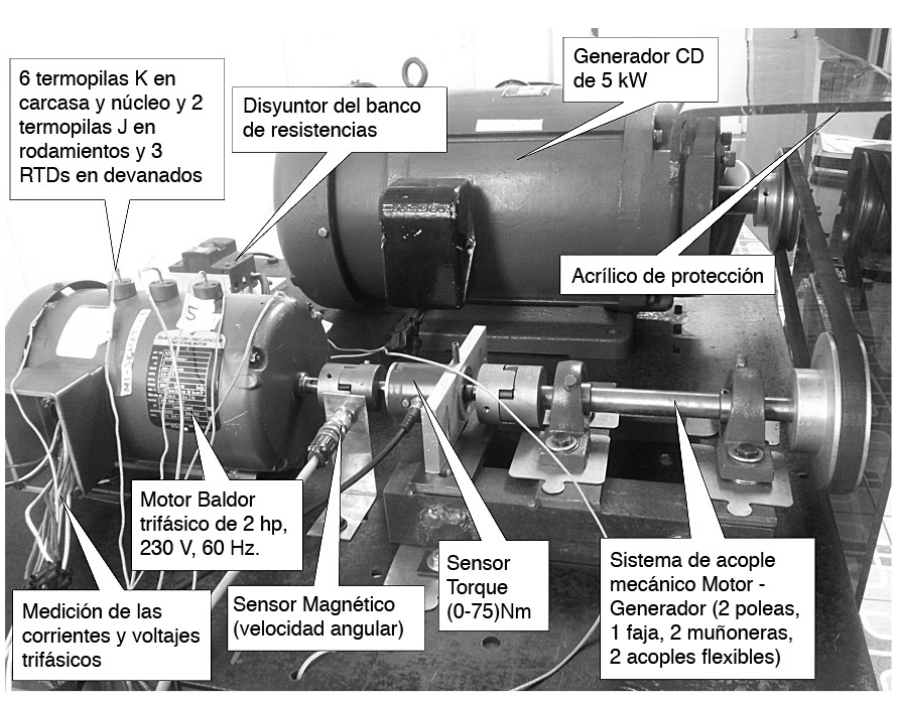

a)

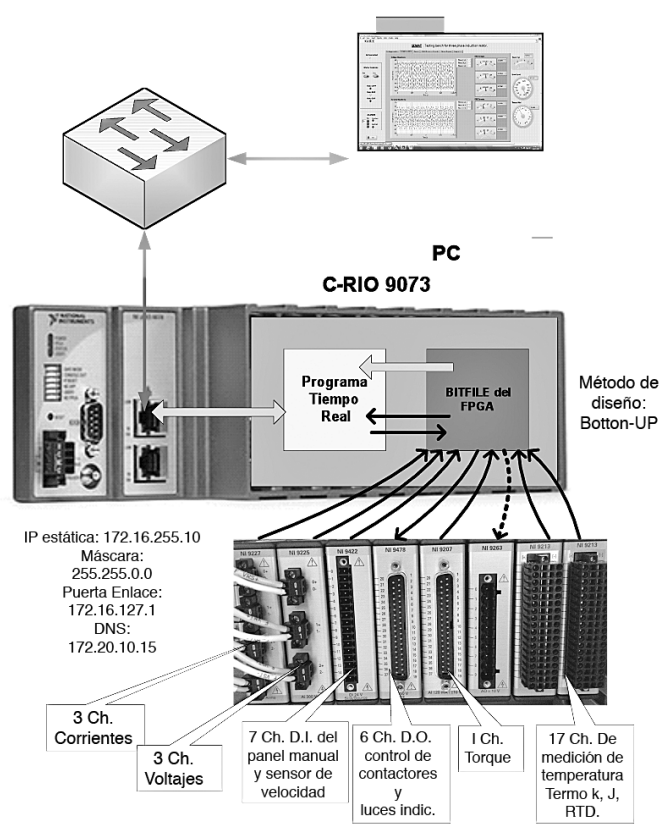

b)

Figura 4. a) Fotografía del banco de pruebas implementado b) Flujo de datos entre la interfaz gráfica y la tarjeta de adquisición de datos, las flechas indican la dirección del flujo de información. Tomado de Murillo-Soto, (2014).

El programa que corre en la PC se conecta mediante una red Ethernet usando el protocolo $\mathrm{TCP} / \mathrm{IP}$ al controlador C-RIO 9073. Este software controla el encendido y apagado del motor seleccionado, muestra las señales trifásicas de corrientes, voltajes y brinda los valores de torque y la velocidad angular del eje. Además muestra en una gráfica las 11 mediciones de 
temperaturas del motor segregadas de la siguiente forma: las temperaturas de cada devanado del motor trifásico, la medición de temperatura de cada rodamiento, dos temperaturas en el núcleo, tres temperaturas en la carcasa y la temperatura ambiental.

Además el software de la PC calcula los valores RMS de corriente y voltaje trifásicos, así como el contenido espectral de las señales. Adicionalmente se calculan los ángulos de fase, las potencias de entrada y salida del motor, calcula y grafica la evolución de la eficiencia de la máquina, calcula el desbalance de la red, el contenido armónico de voltaje y corrientes, así como las potencias reactivas, aparentes y el factor de potencia. Este software permite exportar todas las 28 variables capturadas en dos tipos de archivos de extensión Ivm.

El software que se ejecuta en el C-RIO configura cada uno de los ocho módulos del sistema, su frecuencia de muestro, la sincronización entre módulos de adquisición, el tratamiento de los datos (filtrado), la conversión de datos crudo a variables con significado físico (escalamiento), etc. El controlador manipula salidas digitales para señalización externa, control de contactores y control de un relé de estado sólido mediante modulación de ancho de pulso para el control de la carga eléctrica del generador, que su vez se traduce en carga mecánica en el motor. El software del C-RIO tiene programada la protección de sobrecarga, la protección a un determinado valor de desbalance del voltaje y la protección a valores de contenido armónico superiores a un umbral definido por el usuario.

\section{Cálculo del vector de pérdidas}

El motor en estudio es de la marca Baldor modelo M3558 de 2hp, 230 V, 60 Hz. Lo primero que se realizó son las mediciones de las resistencias estatóricas con un miliohmimetro, el cual arroja los valores por fase de $R_{e}=1.03 \Omega$. Luego se realizó la prueba del motor en vacío la cual permite calcular las perdidas fijas $P_{f}$ ecuación (8), esta prueba consiste en desacoplar la carga mecánica y energizar la máquina con el fin de registrar la potencia de entrada $P_{\text {Ento }}$ y las corrientes de vacío por fase $\left\{I_{A O}, I_{B O}, I_{C O}\right\}$. Con dichas corriente se calcula la pérdida de potencia por efecto Joule en vacío, ecuación (12). Luego se reduce el voltaje y para cada cambio se registran la potencia de entrada en vació y los nuevos valores de corrientes consumida. De esta forma aplicando las ecuaciones (11) y (12) se obtiene la potencia fija para cada voltaje, y se grafica como se muestra en la figura 5. Por lo tanto, las pérdidas de potencias obtenidas para voltaje nominal son: $P_{f}=204,3 W, P_{N}=200,4 W$ y $P_{M}=3,9 W$ respectivamente.

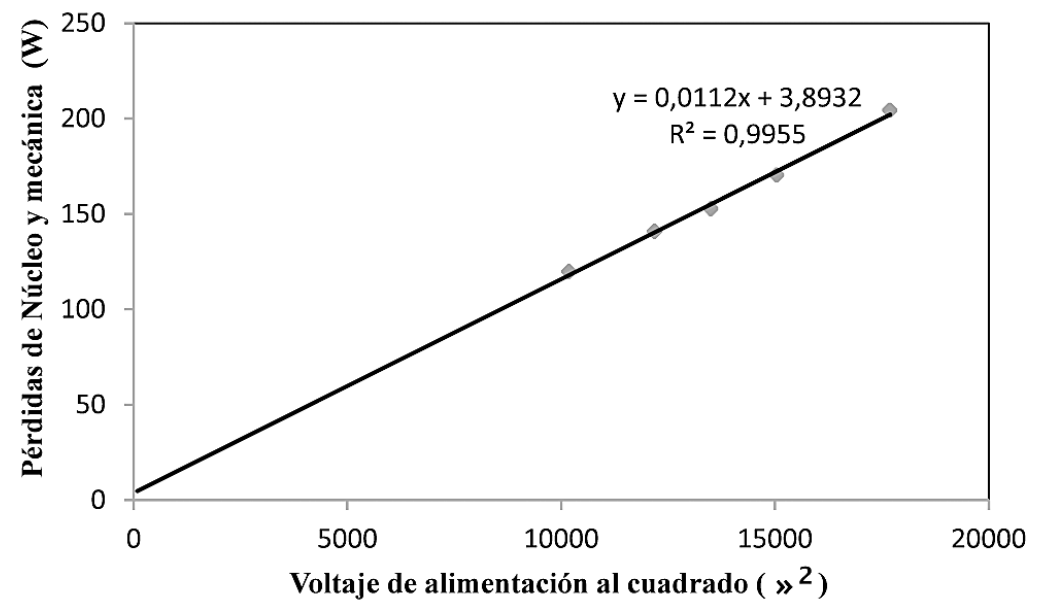

Figura 5. Pérdidas fijas del motor de 2 hp, 230 V, 60 Hz del laboratorio. 
Como tercer paso y dado que el sistema de adquisición de datos calcula las potencias de entrada y salida en cada segundo para una carga dada, se utiliza el promedio de los valores registrados. Por ejemplo la ecuación (13) se modifica y se convierte en la ecuación (20), esto con el fin de utilizar los $N$ registros. El valor calculado fue de $P_{\text {CuE }}=109.4 \mathrm{~W}$.

$$
P_{C u E}=\frac{R_{e}}{N} \sum_{i=1}^{N}\left(I_{A_{i}}{ }^{2}+I_{B i}{ }^{2}+I_{C_{i}}{ }^{2}\right)
$$

Con los valores de pérdidas hasta ahora obtenidos y usando la ecuación (16) se obtiene una potencia de pérdida del estator $P_{s}$, de $309.8 \mathrm{~W}$.

La potencia promedio de entrada es de $P_{\text {Ent }}=1855.6 \mathrm{~W}$ y la potencia promedio de salida es de $P_{\text {Sal }}=1480.3 \mathrm{~W}$, por lo que la potencia total de pérdidas ecuación (7), es de 375.3 W. Para el cálculo de la potencia de pérdidas del rotor se utilizó la expresión (15) ya que se conoce que el deslizamiento promedio es de $3.4 \%$, por lo que $P_{r}=P_{\text {cur }}=52.7 \mathrm{~W}$. La potencia miscelánea $P_{s t}$ para este motor sería de $12.8 \mathrm{~W}$. El vector de pérdidas $P$ del modelo de la ecuación (18) posee por lo tanto los valores: $P=[309.8,52.7]^{\top} \mathrm{W}$.

\section{Identificación de parámetros}

Para estimar las conductancias térmicas $G$ y las capacitancias térmicas $C$ la literatura propone una serie de técnicas por ejemplo: Duran y Fernández, (2004); Moreno, Hidalgo y Martínez, (2001), Boglietti, Cavagnino y Staton, (2008). Alterativamente usando un algoritmo genético es posible estimar dichos parámetros sin mayores complicaciones como se describe en esta sección. Para estimar los parámetros $G=\left[G_{s}, G_{r}, G_{s t}\right]^{T}$ y $C=\left[C_{s}, C_{r}\right]^{T}$ se compara la respuesta $\theta_{\mathrm{s}}$ del modelo de referencia basado en la ecuación (19), con el incremento de temperatura en el estator $\Delta \theta$. El incremento $\Delta \theta$ se entiende como la diferencia entre las temperaturas medidas en el devanado eléctrico $\theta$ y la temperatura ambiente $\Delta \theta=\theta-\theta_{0}$. Los parámetros del modelo de referencia se van ajustando iterativamente mediante un algoritmo genético, de manera tal que la diferencia entre las respuestas de ambos modelos sea mínima.

La figura 6 muestra un diagrama de bloques donde se ilustra dicho proceso. El modelo de referencia se alimenta con el vector de pérdidas $P=\left[P_{s}, P_{r}\right]^{T}$ y los vectores de parámetros a estimar $C=\left[C_{s}, C_{r}\right]^{T}$ y $G=\left[G_{s}, G_{r}, G_{s t}\right]^{T}$ generados por el algoritmo genético. Luego se compara su respuesta con las medidas de temperatura del estator, se ajustan de nuevo los parámetros y se realimenta el modelo. El proceso de optimización se detiene cuando no hay cambios significativos en los vectores de parámetros $C$ y $G$.

La función de incremento de temperatura en el estator $\Delta \theta=\theta-\theta_{0}$ se puede estimar con precisión, pues se tiene la temperatura en el devanado y la temperatura ambiente, las cuales han sido medidas cada segundo a lo largo de un período de 2 horas 30 minutos. De esta forma la función objetivo a optimizar está dada por la función de error cuadrático medio $\varepsilon=\frac{1}{N} \sum_{i=0}^{N}\left(\Delta \theta\left(t_{i}\right)-\theta_{s}\left(t_{i}\right)\right)^{2}$ la cual se obtiene restando los datos experimentalmente medidos $\Delta \theta$ y la respuesta del modelo térmico $\theta_{\mathrm{s}}$.

Para optimizar la función objetivo $\varepsilon$ con el algoritmo genético, se parte de una población inicial de $m=100$ individuos que son generados de forma aleatoria en el espacio de búsqueda. Cada individuo de la población es un vector $X^{k}=\left[C_{s}, C_{r}, G_{s}, G_{r}, G_{s t}\right]^{T}$ para $k=1, \ldots, m$. El espacio de búsqueda se restringió con las siguientes condiciones $0 \leq x^{k}{ }_{i} \leq 100000$ para $i=1, \ldots, 5$.

El operador de cruzamiento combina la información de dos individuos llamados padres para formar, en la siguiente generación, un nuevo individuo llamado hijo. Existen diferentes tipos de 
cruzamiento, se utilizó el llamado cruzamiento heurístico el cual construye el nuevo individuo de la siguiente forma: si $P_{1}$ y $P_{2}$ son los padres tales que $P_{1}$ es el padre mejor adaptado, entonces el nuevo individuo se construye como $\mathrm{H}_{=} \mathrm{P}_{2}+R\left(P_{1}-P_{2}\right)$, donde $R$ es la razón de cruzamiento, en nuestro caso $R=1.2$.

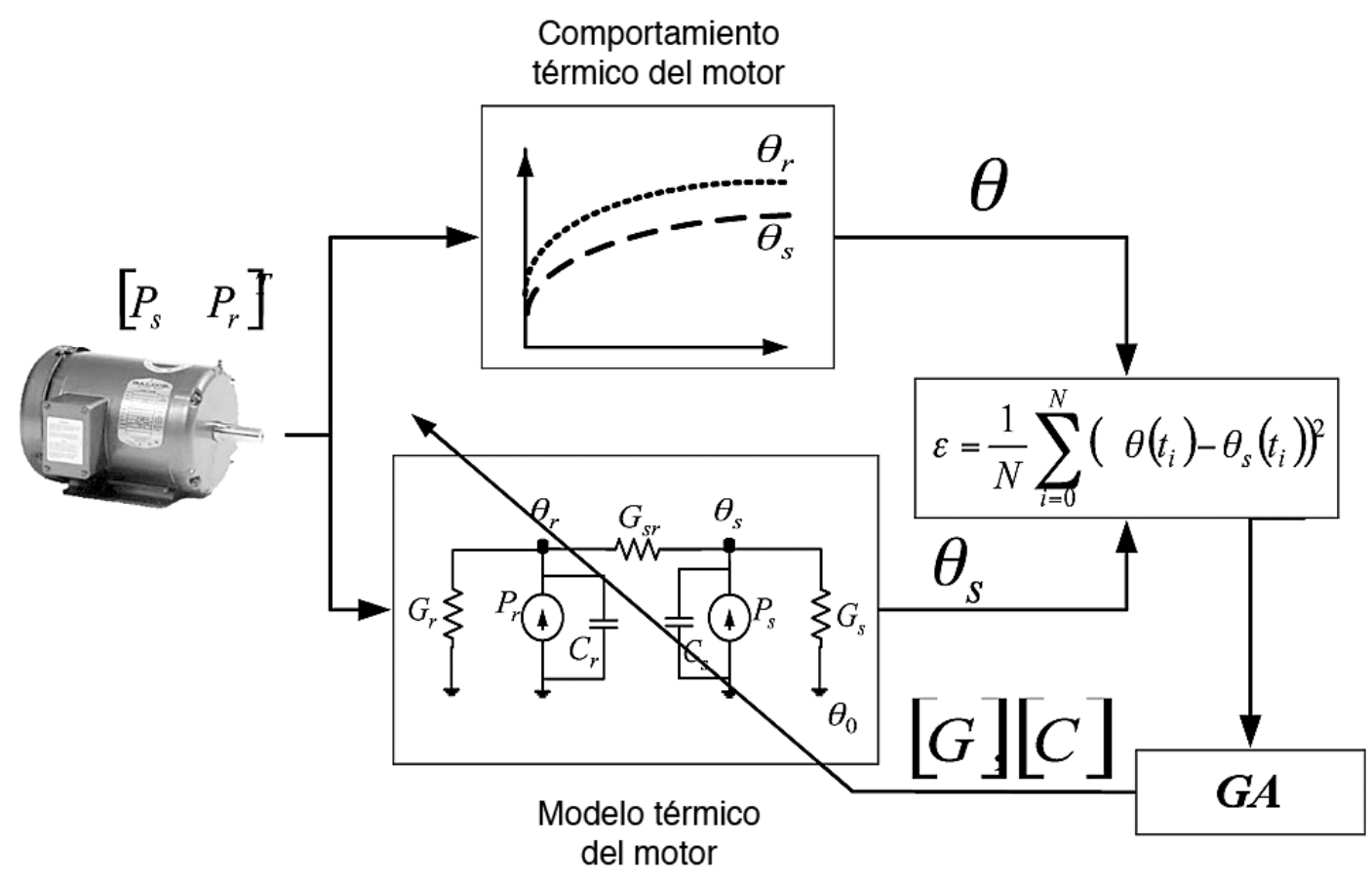

Figura 6. Diagrama de bloques para la identificación de parámetros del modelo térmico.

El operador de mutación realiza aleatoriamente pequeños cambios en los individuos de una población, lo cual provee diversidad genética y amplia el rango de búsqueda. Existen diferentes tipos de mutación, se utilizó la mutación factible adaptativa, la cual genera direcciones de búsqueda aleatorias que son adaptadas de acuerdo al éxito o fracaso de las últimas generaciones. A lo largo de cada dirección se escoge una longitud de avance de modo que se cumplan las restricciones lineales y cotas del problema.

La función de selección elige, tomando en cuenta los valores de aptitud de casa individuo, los padres que se reproducirán para formar la siguiente generación. En nuestro caso la función de selección que se utilizó es el muestreo universal estocástico. Para esta función los individuos son mapeados a un segmento continuo de línea de tal manera que la porción del segmento que se les asigna es proporcional a su aptitud. Se colocan sobre la línea marcas igualmente espaciadas, tantas como padres se quiera seleccionar. El algoritmo se mueve a lo largo de la línea con un paso de avance de igual tamaño. El primer paso de avance es un número aleatorio uniforme menor que las distancias entre las marcas. Los individuos seleccionados son aquellos que están bajo las marcas.

El algoritmo genético se detiene si el cambio acumulado en el valor de la función objetivo a lo largo de las generaciones es menor que la tolerancia de funciones TolFun $=10^{-15}$ o si se alcanza el número máximo de 80000 generaciones que se utilizó. Luego de ejecutar el algoritmo genético para los datos de entrada se obtuvieron los resultados de el cuadro 1. 
Cuadro 1. Parámetros térmicos del motor de $2 \mathrm{Hp}, 230 \mathrm{~V}, 60 \mathrm{~Hz}$.

\begin{tabular}{|c|c|}
\hline Símbolo & Valor \\
\hline$C_{s}$ & $5243.41 \mathrm{~J} /{ }^{\circ} \mathrm{C}$ \\
\hline$C_{r}$ & $5389.43 \mathrm{~J} /{ }^{\circ} \mathrm{C}$ \\
\hline$G_{s}$ & $4.15 \mathrm{~W} /{ }^{\circ} \mathrm{C}$ \\
\hline$G_{r}$ & $3 \times 10^{\wedge}-8 \mathrm{~W} /{ }^{\circ} \mathrm{C}$ \\
\hline$G_{s r}$ & $12.16 \mathrm{~W} /{ }^{\circ} \mathrm{C}$ \\
\hline Mejor aptitud & 0.5317 \\
\hline Aptitud promedio & 0.5350 \\
\hline
\end{tabular}

\section{Validación}

El motor de estudio posee una masa de $16 \mathrm{~kg}$ de los cuales $10 \mathrm{~kg}$ pertenecen al estator y carcasa y el resto al rotor y al eje. Una primera aproximación para ver el orden de magnitud de las capacitancias es aplicar las fórmulas:

$$
\begin{aligned}
& C_{s}=m_{s, i r} \cdot c_{s, i r}+m_{s, c u} \cdot c_{s, c u} \\
& C_{r}=m_{r, i r} \cdot c_{s, i r}+m_{r, a l} \cdot c_{s, c u}
\end{aligned}
$$

donde la masa del acero del estator $m_{s, i r}$ es de $8 \mathrm{~kg}$, la masa del cobre en el estator $m_{s, c u}$ es de $2 \mathrm{~kg}$, la masa del acero en el rotor $m_{r, i r}$ es $5,4 \mathrm{~kg}$ y la masa del aluminio en el rotor es de 0,6 kg. Los calores específicos del acero, cobre y aluminio son $[0.50 .390 .9]^{T} \mathrm{~J} /\left(g^{\circ} \mathrm{C}\right)$ respectivamente. Aplicando (21) y (22) el vector $\left[C_{s} C_{r}\right]^{T}$ es igual a $[47803240]^{T} \mathrm{~J} /{ }^{\circ} \mathrm{C}$.

Las resistencias térmicas se calcularon usando las fórmulas que propone Boglietti, Cavagnino, Lazzari, y Pastorelli, (2003), donde el cálculo de las resistencias térmicas se realiza a partir del conocimiento de las mediciones internas del motor, los materiales y constantes universales. Con esto se plantea la descomposición del motor en varios cilindros concéntricos y se calcula la resistencia radial para cada cilindro. El cuadro 2 muestra los resultados de las resistencias térmicas del modelo de la figura 7.

\begin{tabular}{|c|c|c|}
\hline$R_{e c a}$ & $0.5116^{\circ} \mathrm{C} / \mathrm{W}$ & Resistencia por convección forzada entre la carcasa y el ambiente \\
\hline$R_{0}$ & $0.3063^{\circ} \mathrm{C} / \mathrm{W}$ & Resistencia por convección natural entre la carcasa y el ambiente \\
\hline$R_{s y 1}$ & $0.0041^{\circ} \mathrm{C} / \mathrm{W}$ & Resistencia por conducción radial de la parte baja del núcleo \\
\hline$R_{\text {sy } 2}$ & $0.0041^{\circ} \mathrm{C} / \mathrm{W}$ & Resistencia por conducción radial de la parte alta del núcleo \\
\hline$R_{s t}$ & $0.0291^{\circ} \mathrm{C} / \mathrm{W}$ & Resistencia por conducción de diente del estator \\
\hline$R_{c u, i r}$ & $0.01796^{\circ} \mathrm{C} / \mathrm{W}$ & Resistencia por conducción entre cobre y el diente del núcleo \\
\hline$R_{e w, e c}$ & $4.2265^{\circ} \mathrm{C} / \mathrm{W}$ & Resistencia por conducción entre devanado externo y carcasa \\
\hline$R_{\text {airgap }}$ & $0.0888^{\circ} \mathrm{C} / \mathrm{W}$ & Resistencia del entrehierro \\
\hline$R_{\text {shf }}$ & $37.4184^{\circ} \mathrm{C} / \mathrm{W}$ & Resistencia axial por conducción del eje \\
\hline$R_{\text {sig }}$ & $0^{\circ} \mathrm{C} / \mathrm{W}$ & Resistencia entre el núcleo y la carcaza \\
\hline$R_{i a, e c}$ & $\infty^{\circ} \mathrm{C} \mathrm{W}$, Sin datos & Resistencia por convección entre aire interno y tapas \\
\hline$R_{\text {ew,ia }}$ & $\infty^{\circ} \mathrm{C} / \mathrm{W}$, Sin datos & Resistencia por convección entre devanado externo y aire interno \\
\hline
\end{tabular}

Cuadro 2. Valores de las resistencias térmicas obtenidas según Boglietti et al., (2003) 


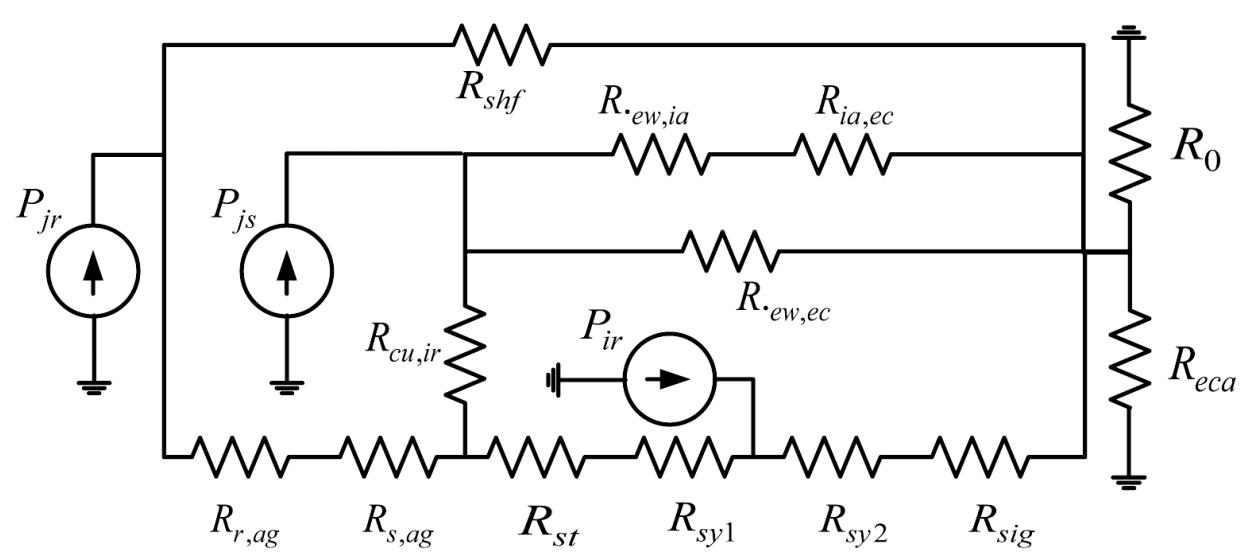

Figura 7. Modelo térmico propuesto por Boglietti et al., (2003).

A partir del análisis del modelo de la figura 7, se plantean las ecuaciones para determinar las conductancias térmicas del modelo simplificado representado por (18).

$$
\begin{gathered}
G_{s}=\frac{1}{\left(\frac{1}{R_{c u, i r}+R_{s t}+R_{s y 1}+R_{s y 2}}+\frac{1}{R_{e w, e c}}+\frac{1}{R_{i a, e c}+R_{e w, i a}}\right)^{-1}+\left(\frac{1}{R_{0}}+\frac{1}{R_{e c a}}\right)^{-1}} \\
G_{s r}=\frac{1}{R_{r, a g}+R_{r, 1, g}}=\frac{1}{R_{\text {airgap }}} \\
G_{r}=\frac{1}{R_{s h f}+\left(\frac{1}{R_{0}}+\frac{1}{R_{e c a}}\right)^{-1}}
\end{gathered}
$$

Las conductancias térmicas $G_{s}, G_{r}, G_{s r}$ se calculan usando las tres ecuaciones anteriores y los datos del cuadro 2. Los valores calculados son $G=\left[\begin{array}{llll}4.06 & 0.026 & 11.26\end{array}\right]^{\top} \mathrm{W} /{ }^{\circ} \mathrm{C}$.

\section{Análisis de resultados}

El cálculo de las capacitancias y conductancias térmicas realizadas en la sección anterior responden a una aproximación que utiliza las dimensiones del motor, sus materiales y constantes físicas, etc. Por lo tanto, es esperable que existan errores dado que no se conocían algunas constantes constructivas del motor y se debieron estimar según la literatura (Boglietti et al., 2003). Los parámetros obtenidos sirven para comparar el orden de las magnitudes con los resultados del algoritmo genético. Los errores obtenidos entre los parámetros del algoritmo genético y los parámetros calculados por las ecuaciones de la (23) a la (25) se muestra en el cuadro 3.

Cuadro 3. valor absoluto del error en los Parámetros térmicos

\begin{tabular}{|c|c|}
\hline Símbolo & Error relativo al A.G \\
\hline$C_{s}$ & $8.8 \%$ \\
\hline$C_{r}$ & $39.8 \%$ \\
\hline$G_{s}$ & $2.2 \%$ \\
\hline$G_{r}$ & $100 \%$ \\
\hline$G_{s r}$ & $7.4 \%$ \\
\hline
\end{tabular}


Se observa que existen dos parámetros con errores altos $C_{r}$ y $G_{r}$ ambos relacionados con el rotor. La capacitancia del rotor obtenida por el algoritmo genético es mayor que la obtenida por la ecuación (22), esto se debe principalmente a que esta ecuación no contempla el almacenamiento de calor del aire confinado en motores sellados como el motor en estudio, por lo que si se contemplara este hecho en dicha ecuación, la capacitancia tendería a ser mayor y, por tanto, a asemejarse a la obtenida por el GA. Por otra parte la conductancia térmica del rotor obtenida por el GA indica que es prácticamente cero, lo cual se interpreta como que el calor producido por el rotor fluye a través del entrehierro y no por el eje del motor hacia el ambiente. Este resultado si concuerda por el obtenido con la ecuación (25) que da un valor de 0,026 $\mathrm{W} /{ }^{\circ} \mathrm{C}$, sin embargo cuando se calcula el error relativo este da un valor de $100 \%$, esto se debe a que cualquier número respecto a un valor de casi cero tendrá un error muy alto como en este caso.

El modelo obtenido por las ecuaciones (19) es alimentado con los parámetros obtenidos por el GA cuadro 1 y por los parámetros analíticos obtenidos por las ecuaciones (21) a la (25). Ambos conjuntos de parámetros generan para el mismo modelo respuestas dinámicas distintas tal y como se aprecia en la figura 8, obteniéndose unas diferencias máximas de las respuestas de temperaturas de $11^{\circ} \mathrm{C}$ en el trasciende y de $5^{\circ} \mathrm{C}$ en estado estable.

Finalmente, se rescata que el modelo térmico con los parámetros obtenidos por el GA presenta un mejor ajuste respecto a los datos experimentales $\varepsilon=0.5317$, que el modelo con parámetros analíticos con ajuste de $\varepsilon=50.65$, el ajuste del algoritmo genético se observa en la figura 9 . Esto, como se dijo anteriormente se debe a que el algoritmo genera 80000 conjuntos de valores posibles y selecciona un conjunto con la mejor aptitud. Por lo tanto, el método para obtener los parámetros de un modelo térmico simple con algoritmos genéticos, es una opción eficiente en el sentido de que no se requiere conocer características físicas, materiales, etc., del motor en estudio.

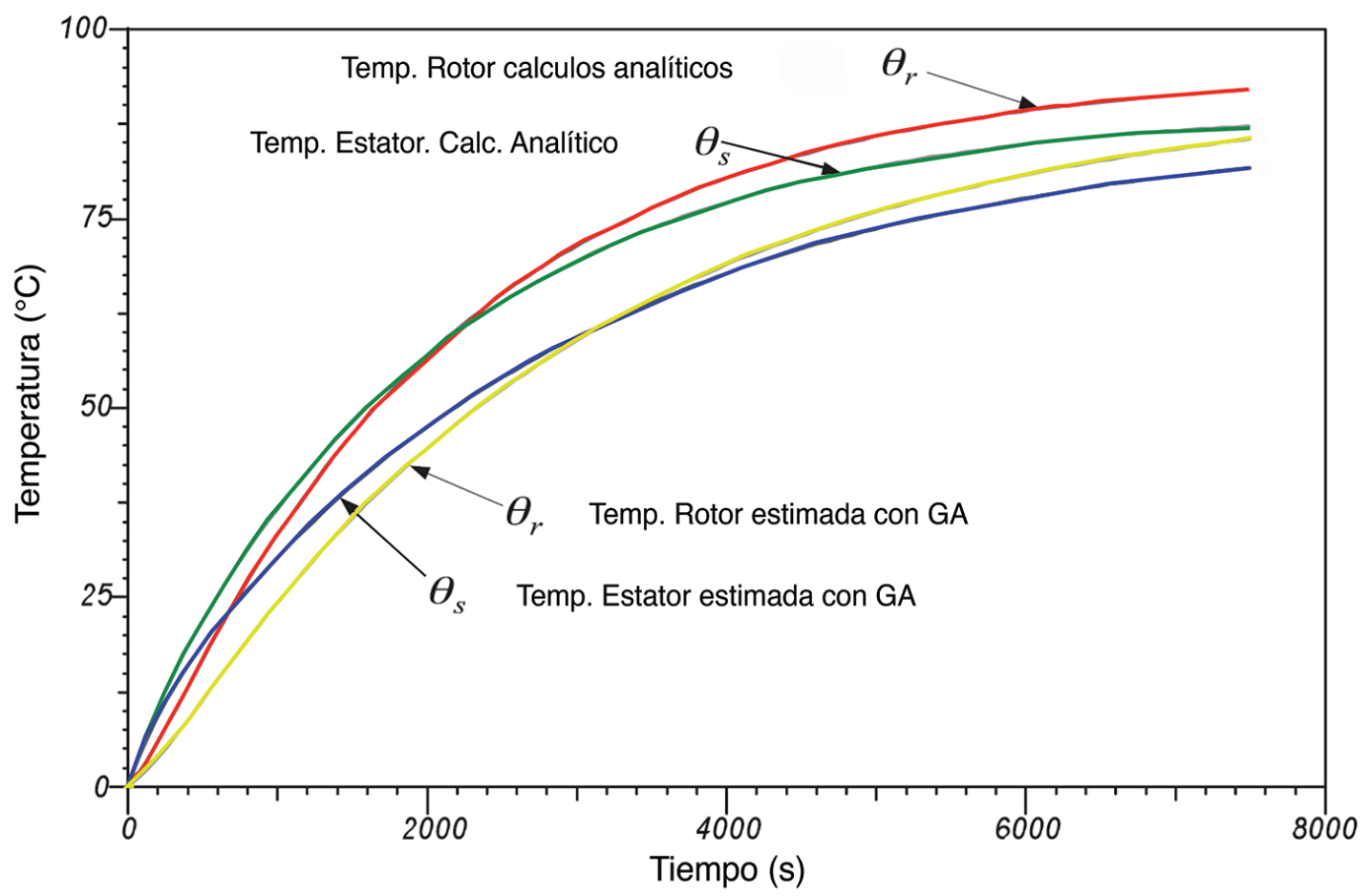

Figura 8. Simulación de las temperaturas del rotor y estator para el modelo planteado (19) según los parámetros obtenidos por algoritmo genético y los obtenidos de forma analítica. 


\section{Agradecimiento}

Se agradece a la Vicerrectoría de Investigación del Instituto Tecnológico de Costa Rica por el financiamiento brindado al proyecto SEMAT, código VIE 5402-1341-1301, así como a los asistentes vinculados a este proyecto.

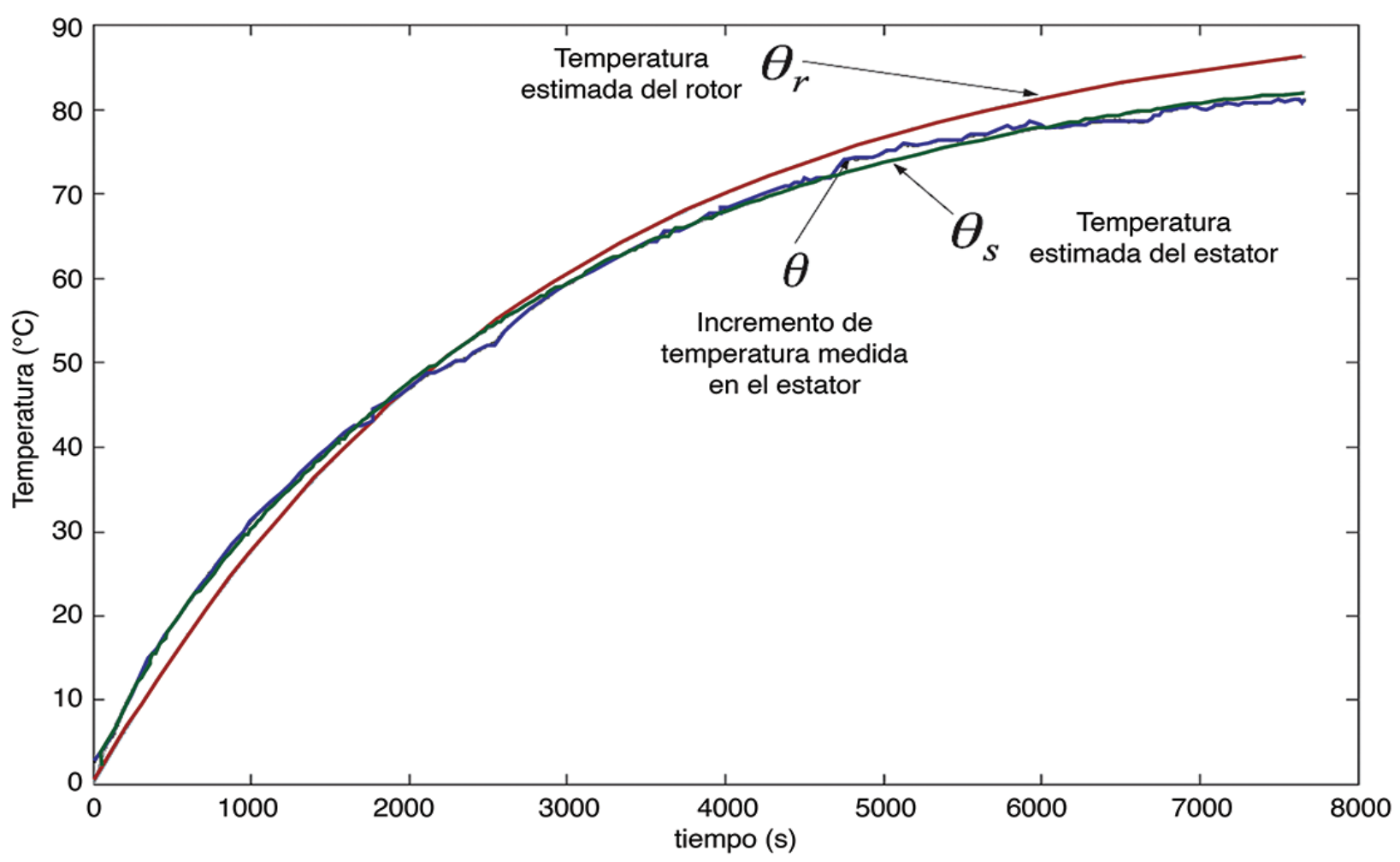

Figura 9. Ajuste de temperaturas entre la medida y la estimada.

\section{Bibliografía}

Boglietti, A., Cavagnino, A., Lazzari, M., \& Pastorelli, M. (2003). A Simplified Thermal Model for Variable-Speed Self-Cooled Industrial Induction Motor. IEEE Transactions on Industry Applications, 39(4), 945-952. http://doi. org/10.1109/TIA.2003.814555

Boglietti, A., Cavagnino, A., \& Staton, D. (2008). Determination of Critical Parameters in Electrical Machine Thermal Models. IEEE Transactions on Industry Applications, 44(4), 1150-1159. http://doi.org/10.1109/TIA.2008.926233

Boldea, I., \& Nasar, S. A. (2002). The Induction Machine Handbook. CRC Press.

Cho, K., \& Seok, J. (2008). Induction Motor Temperature Estimation Based on High- Frequency Model of Rotor Bar. In Industry Applications Society Annual Meeting, 2008. IAS '08. IEEE (pp. 1-7). http://doi. org/10.1109/08IAS.2008.208

Duran, M. J., \& Fernandez, J. (2004). Lumped-Parameter Thermal Model for Induction Machines. IEEE Transactions on Energy Conversion. http://doi.org/10.1109/TEC.2004.837272

Dutta, B., \& Chowdhury, S. K. (2012). Steady state thermal model of TEFC induction machine. In IEEE International Conference on Emerging Technologies and Factory Automation, ETFAPower Electronics, Drives and Energy Systems (PEDES), 2012 (pp. 1-6). Bengaluru. http://doi.org/10.1109/PEDES.2012.6484344

Fraile-Mora, J. (2008). Máquinas Eléctricas (6th ed.). España: McGraw-Hill Interamericana de España. 
Gao, Z. (2006). SENSORLESS STATOR WINDING TEMPERATURE ESTIMATION FOR INDUCTION MACHINES. Georgia Institute of Technology.

Gao, Z., Habetler, T. G., Harley, R. G., \& Colby, R. S. (2005). An Adaptive Kalman Filtering Approach to Induction Machine Stator Winding Temperature Estimation Based on a Hybrid Thermal Model. IEEE Industry Applications Conference, 2-9. http://doi.org/10.1109/IAS.2005.1518284

IEEE Standard Test Procedure for Polyphase Induction Motors and Generators. (2004). IEEE Std 112-2004 (Revision of IEEE Std 112-1996), 0_1-79. http://doi.org/10.1109/ieeestd.2004.95394

Kim, D. J., Choi, J. H., Chun, Y. Do, Koo, D. H., \& Han, P. W. (2014). The study of the stray load loss and mechanical loss of three phase induction motor considering experimental results. Journal of Electrical Engineering and Technology, 9, 121-126. http://doi.org/10.5370/jeet.2014.9.1.121

Kylander, G. (1995). Thermal modelling of small cage induction motors. Chalmers University of Technology. Retrieved from http://publications.lib.chalmers.se/records/fulltext/1335/1335.pdf

Moreno, J. F., Hidalgo, F. P., \& Martínez, M. D. (2001). Realisation of tests to determine the parameters of the thermal model of an induction machine. IEE Proceedings - Electric Power Applications. http://doi.org/10.1049/ ip-epa:20010580

Murillo-Soto, L. D. (2014). Aplicación informática para el monitoreo de variables eléctricas, mecánicas y térmicas en motores trifásicos de inducción. In Latin American and Caribbean Conference for Engineering and Technology (LACCEl'2014). Guayaquil: EBSCO. Retrieved from http://www.laccei.org/LACCEI2014-Guayaquil/ ExtendedAbstracts/EA026.pdf

Okoro, O. I., Weidemann, B., \& Ojo, O. (2004). An efficient thermal model for induction machines. Conference Record of the 2004 IEEE Industry Applications Conference, 2004. 39th IAS Annual Meeting., 4. http://doi.org/10.1109/ IAS.2004.1348823

Oraee, H. (2000). A quantative approach to estimate the life expectancy of motor insulation systems. IEEE Transactions on Dielectrics and Electrical Insulation, 7(6), 790-796. http://doi.org/10.1109/94.891990

Pillay, P., \& Manyage, M. (2006). Loss of life in induction machines operating with unbalanced supplies. IEEE Transactions on Energy Conversion, 21(4), 813-822. http://doi.org/10.1109/TEC.2005.853724

Rudolph, G. (1994). Convergence analysis of canonical genetic algorithms. IEEE Transactions on Neural Networks, 5 , 96-101. http://doi.org/10.1109/72.265964

Shenkman, a. L., \& Chertkov, M. (2000). Experimental method for synthesis of generalized thermal circuit of polyphase induction motors. IEEE Transactions on Energy Conversion, 15(3), 264-268. http://doi.org/10.1109/60.875491

Talbi, E. G. (2009). Metaheuristics: From Design to Implementation. Metaheuristics: From Design to Implementation. http://doi.org/10.1002/9780470496916

Zocholl, S. E. (2004). Comparing motor thermal models. In In 31st Annual Western Protective Relay Conference (pp. 1-10). WA.

Zocholl, S. E., Schweitzer, E. O., \& Aliaga-Zegarra, A. (1984). Thermal Protection of Induction Motors Enhanced by Interactive Electrical and Thermal Models. IEEE Transactions on Power Apparatus and Systems, PAS-103(7), 1749-1755. http://doi.org/10.1109/TPAS.1984.318678 